\title{
A group contribution method for predicting the solubility of mercury
}

\author{
Mansour Khalifa ${ }^{\mathrm{a}, *}$, Leo Lue ${ }^{\mathrm{a}}$ \\ ${ }^{a}$ Department of Chemical and Process Engineering, University of Strathclyde, James Weir Building, 75 Montrose Street, Glasgow G1 1XJ, \\ United Kingdom
}

\begin{abstract}
Mercury is a toxic and corrosive element, and understanding its partitioning within ecosystems and industrial processes is of vital importance. The solubility of mercury in normal alkanes, aromatics, water and alcohols is predicted using widely used Soave-Redlich-Kwong equation of state in combination with a group contribution method to estimate binary interaction parameters. The interaction parameters between elemental mercury and seven other molecular groups were determined in this work by fitting available solubility data for mercury. The solubility in the studied solvents was accurately described. This work allows the prediction of the thermodynamic behavior of elemental mercury in a wide variety of solvents, solvent mixtures, and operating conditions where experimental data are unavailable.
\end{abstract}

Keywords: mercury, solubility, equation of state, group contribution method

\section{Introduction}

Mercury occurs naturally in the environment and can be found in soil, air, and water. Due to its toxicity and accumulative nature, it is considered a highly dangerous element $[1,2,3]$. The sources of mercury in the biosphere can be divided into natural and anthropogenic sources. Both are considered to be equally important causes of mercury accumulation in the environment. Natural sources include volcanic activity, erosion of terrain, and dissolution of mercury minerals in the oceans, lakes and rivers [4]. Anthropogenic sources include cement manufacturing, paper milling, the combustion of coal, oil, and gas as fuel to generate power, flared gas from onshore and offshore oil and gas platforms, and produced water discharged from oil and gas processing facilities, refineries and chemical plants $[5,6,7]$.

In addition to its contribution to environmental pollution, mercury has a negative impact on the production and processing of oil and gas. As mercury is present in many major oil fields, maintenance and operation teams can be exposed to this highly dangerous element on a daily basis. Activities that expose workers to mercury include equipment cleaning, oil sampling, vessel and tank inspections and hot work activities on restricted areas. The risks are proportional to the concentration

\footnotetext{
${ }^{*}$ Corresponding author

Email addresses: khalifa.manssor@strath.ac.uk (Mansour Khalifa), leo.lue@strath.ac.uk (Leo Lue)

of mercury in the process facilities [8]. Mercury not only poses health risks, it is also corrosive and can cause equipment degradation and damage, catalyst poisoning, etc. [9]. It has an ability to accumulate on primary and secondary process treatment units (e.g., amine units, glycol units, cryogenic units and heat exchangers), eventually causing process failure [8].

In general, there are three ways to avoid mercury emissions from industrial sources to the environment: preventive measures, primary control measures and secondary control measures. Preventive measures can be achieved only if the source of emissions is prevented; this means fuel substitution, such as use of renewable energy or biofuels instead of mercury containing energy sources. However, this option is not popular as oil and gas are still the main source of energy. In primary control measures, $\mathrm{Hg}$ emissions are still generated but reduced to a certain value. In secondary control measures, $\mathrm{Hg}$ emissions exist but are removed later from exhaust gases [10]. The selection of the best measures requires a good understanding of the behavior of mercury and its pathways through the process and the environment. Consequently, mercury exposure risks can be mitigated by determining the concentration and understanding the exposure pathways in work locations and adopting effective health and safety policies and procedures accordingly. Understanding mercury pathways in industrial processes and the ecosystem requires knowledge of the thermodynamic behavior of mercury and its

October 25, 2016 
interactions with other compounds, such as process fluids (e.g., water, hydrocarbon mixtures, etc.).

Many physical properties of mercury, such as density, thermal expansion and compressibility as a function of temperature and pressure have been measured [11]. Vapor pressure is one of the most important properties, as it indicates the state of mercury and its concentration in vapor, liquid, and solid phases. Several experimental measurements and correlations have been published for the calculation of mercury vapor pressure over wide range of temperatures $[12,13]$.

Predicting the solubility of mercury in liquids and gases gives an indication of mercury pathways from one phase to another. Accurate prediction of mercury solubility plays an important role in developing a risk mitigation strategy. In general, however, the available experimental data are limited, in part, due to the difficulty in working with mercury. Experiments involving mercury can be time consuming and costly, and it is difficult to anticipate the wide range of process conditions and fluids that may be encountered.

In situations where experimental data are unavailable, predictive methods are required. A competitive model should be computationally inexpensive to evaluate and require minimal parameterization [14]. One powerful method is molecular simulation, which requires force fields to be parameterized between all species in the solution. This work has been focused particularly on systems containing elemental mercury [15] and some mercury compounds $[16,15]$ in water. While these methods offer the possibility of predicting thermodynamic properties of system containing mercury, they are computationally intensive and not suitable for use in process scale simulations (e.g., in a refinery).

Another approach is to use thermodynamic models, such as an equations of state (EOS) or an activity coefficient model [17]. These models have been successfully used for the estimation of physical and chemical properties of pure and multicomponent systems. The selection of a model depends on its capability of estimating the required physical and chemical properties, and predicting the phase behavior of a specific system where experimental data are unavailable. Equations of state, such as cubic equations of state or the perturbed-chain statistical associating fluid theory (PCSAFT), are characterized by their simplicity, reliability and robustness over a wide range of conditions (e.g., high pressures), and speed of computation [18]. Therefore, they are the model of choice for many multicomponent systems and are widely used for practical applications.

A recent study used PCSAFT to describe the phase behavior of elemental mercury in liquid and compressed hydrocarbon gases [19]. While this approach works well, it requires fitting binary interaction parameters $k_{i j}$ between mercury and the specific solvents being modeled to existing experimental measurements. Properly accounting for the interactions between molecules is vital to the accurate prediction of mixture properties. Within the context of equations of state, this is typically achieved by introducing the binary interaction parameter $k_{i j}$ between different molecular species; $k_{i j}$ is normally used as a fitting parameter that is adjusted to minimize the differences between the calculated and experimentally measured system properties, such as VLE, LLE, density, and solubility. This limits its usage to solvents where measurements with mercury exist.

One approach developed in order to overcome this issue is the group contribution method (GCM). In the GCM, molecules are subdivided into a series of groups which consist of individual atoms or collections of atoms [20]. The binary interaction parameters between two molecules is then given as the sum of the interaction parameters between the various pairs of groups on each of the molecules. This allows the prediction of $k_{i j}$ for a large number of compounds where experimental data are unavailable and at operating conditions outside the range of measurements.

In this work, we parameterize a group contribution method to estimate the binary mixing parameters for the Soave-Redlich-Kwong (SRK) equation of state to the estimation of the thermodynamic properties of elemental mercury in mixtures of water, alkanes, aromatics, and alcohols. The SRK EOS is used in this work because of its simplicity, computational efficiency, and ability to predict vapor-liquid equilibria (VLE) and liquid-liquid equilibria (LLE) at high and low pressures. The $k_{i j}$ of elemental mercury was predicted using the GCM developed by Peneloux and co-workers [20]. This has the advantage that the group interaction parameters already exist [21, 22, 23] for the SRK EOS for a wide range of molecular groups, and so the method can be immediately used in practical calculations. Combined with a group contribution method, the SRK EOS allows the prediction of mercury solubility and partitioning between phases.

In the next section, we briefly review the SRK EOS and its application to mixtures. We then present the group contribution method that is used to predict the binary interaction parameters required by the equation of state. In Sec. 3, this theory is compared against experimental measurements for the solubility of mercury in water, $n$-alkanes, aromatic solvents, and alcohols. Finally, the main points of this paper are summarized in Sec. 4, and directions for future work are discussed. 


\section{Methodology}

The SRK EOS is a modification of a cubic equation of state proposed by Redlich and Kwong [24] developed by Soave [25] by studying the behavior of pure compounds:

$$
p=\frac{\rho R T}{1-\rho b}-\frac{a \rho^{2}}{(1+\rho b)}
$$

where $p$ is the system pressure, $T$ is the absolute temperature, and $R$ is the universal gas constant, $\rho$ is the molar density of the system, and $a$ and $b$ are parameters of the model. The first term of Eq. (1) corresponds to the repulsive force and the second term corresponds to the attraction force. The parameters $a_{i}$ and $b_{i}$ for a pure component $i$ can be expressed in terms of its critical temperature $T_{c i}$ and critical pressure $p_{c i}$

$$
\begin{aligned}
a_{i} & =0.42747 \frac{R^{2} T_{c i}^{2}}{p_{c i}} \alpha_{i}(T) \\
\alpha_{i}(T) & =\left[1+\left(0.480+1.57 \omega_{i}-0.176 \omega_{i}^{2}\right)\left(1-\sqrt{\frac{T}{T_{c i}}}\right)\right]^{2} \\
b_{i} & =0.08664 \frac{R T_{c i}^{2}}{p_{c i}}
\end{aligned}
$$

where $\omega_{i}$ is the acentric factor for component $i$, introduced by Pitzer [17].

To extend the SRK EOS to multi-component systems, mixing rules are required to obtain the parameters $a$ and $b$ for the solution from the $a_{i}$ 's and $b_{i}$ 's from the individual pure components. Many mixing rules have been proposed for cubic EOS [26, 27]. In this work, we use the van der Waals mixing rules, which are given by

$$
\begin{aligned}
& a=\sum_{i j} x_{i} x_{j} \sqrt{a_{i} a_{j}}\left(1-k_{i j}\right) \\
& b=\sum_{i} x_{i} b_{i}
\end{aligned}
$$

where $k_{i j}$ in Eq. (5) is the binary interaction parameter, $x_{i}$ is the mole fraction of component $i$ in the mixture, and $a_{i}$ and $b_{i}$ are calculated from Eqs. (2) and (4).

\subsection{The Helmholtz Energy}

From knowledge of the Helmholtz energy as a function of the temperature, volume, and component densities, all thermodynamic properties of a system can be determined. For an ideal gas, the molar Helmholtz en$\operatorname{ergy} A^{i g}$ is given by

$$
A^{i g}(T, \rho)=\sum_{i=1} x_{i} \mu_{i}^{\circ}+R T \sum_{i=1} x_{i}\left(\ln \rho_{i} b_{i}-1\right)
$$

where $\rho_{i}$ is the molar density of component $i$, and $\mu_{i}^{\circ}$ is the standard state chemical potential.

In order to consider real systems, we define a residual property as the difference between the property of the actual system and that of an ideal gas at the same total volume, temperature and number of moles of each species [18]

$$
M^{r e s}(T, V, n)=M(T, V, n)-M^{i g}(T, V, n)
$$

where $M^{\text {res }}$ is any residual property.

The molar residual Helmholtz energy $A^{\text {res }}$ can be directly determined from the equation of state by

$$
A^{r e s}=R T \int_{0}^{\rho} \frac{d \rho}{\rho}(Z-1)=I
$$

where $Z=p /(\rho R T)$ is the compressibility factor, and $\rho$ is the molar density of the system.

The compressibility factor for the SRK equation of state can be written as:

$$
\begin{aligned}
Z & =\frac{1}{1-\rho b}-\frac{a}{b^{2} R T} \frac{\rho b}{1+\rho b} \\
& =Z_{\text {exc }}+Z_{\text {att }}
\end{aligned}
$$

where $Z_{\text {exc }}$ accounts for excluded volume interactions, and $Z_{\text {att }}$ accounts for attractive interactions. Substituting Eq. (10) into Eq. (9), $A^{\text {res }}$ can be written as:

$$
A^{r e s}=I_{e x c}(\rho b)-E(T, x) Q(\rho b)
$$

where $I_{\text {exc }}$ is the contribution from excluded volume interactions, $E$ characterizes the dependence of the attractive interactions in the system on the composition and temperature, and $Q$ captures the influence of density (which is related to the "frequency" of the interactions). For the SRK EOS, these terms are explicitly given by

$$
\begin{aligned}
I_{e x c}(\rho b) & =-R T \ln (1-\rho b), \\
E(T, x) & =\frac{a}{b^{2}}, \\
Q(\rho b) & =\ln (1+\rho b) .
\end{aligned}
$$

In order to characterize the influence of mixing on a system, we first define an ideal solution, where the Helmholtz energy is defined as:

$$
A^{i d}=\sum_{i=1} x_{i} A_{i}^{\circ}+R T \sum_{i=1} x_{i} \ln x_{i}
$$

where $A_{i}^{\circ}$ is the molar Helmholtz energy of pure component $i$, which is given by

$$
A_{i}^{\circ}=\mu_{i}^{\circ}+R T\left(\ln \rho b_{i}-1\right)+I_{i}^{\circ}
$$


where $I_{i}^{\circ}$ is the molar residual Helmholtz free energy of pure $i$ at packing fraction $\rho b_{i}$ and temperature $T$. We also define an excess property as the difference between the actual value of the property of the system and the value of an ideal mixture at the same temperature, total moles of each species and packing fraction [18]:

$$
M^{E}(T, n, \rho b)=M(T, n, \rho b)-M^{i d}(T, n, \rho b)
$$

where $M^{E}$ is the excess property and $M^{i d}$ is the ideal mixture property.

The excess Helmholtz free energy at constant temperature, constant volume, and constant number of moles of each species can be defined based on Eq. (17) as:

$$
\begin{aligned}
A^{E}(T, n, \rho b) & =A(T, n, \rho b)-A^{i d}(T, n, \rho b) \\
& =R T \sum_{i} x_{i} \ln \frac{b_{i}}{b}+I-\sum_{i} x_{i} I_{i}^{\circ} .
\end{aligned}
$$

The first term represents effect of molecule size on the free energy of mixing, while the final two terms give the influence of the attractive interactions between molecules.

For an equation of state similar in form to the SRK EOS, the excess Helmholtz free energy can be written as

$$
A^{E}(T, n, \rho b)=R T \sum_{i} x_{i} \ln \frac{b_{i}}{b}+\frac{Q(\rho b)}{2 b} \sum_{i, j} x_{i} x_{j} b_{i} b_{j} E_{i j}
$$

where $E_{i j}$ physically captures the free energy of interaction between a molecule of type $i$ and a molecule of type $j$, and the $Q$ term describes the frequency of the interactions. The parameter $E_{i j}$ can be directly related to the original parameters of the SRK equation of state as

$$
E_{i j}=-2 \frac{a_{i j}}{b_{i} b_{j}}+\frac{a_{i}}{b_{i}^{2}}+\frac{a_{j}}{b_{j}^{2}}
$$

Using the van der Waals mixing rules (see Eqs. (5) and (6)) leads to:

$$
E_{i j}=\left(\delta_{i}-\delta_{j}\right)^{2}+2 \delta_{i} \delta_{j} k_{i j}
$$

where $\delta_{i}=a_{i}^{1 / 2} / b_{i}$ is the Scatchard-Hildebrand solubility parameter $[17,22,28]$. So we see that the binary interaction parameter $k_{i j}$ describes the deviation of the interaction free energy between two molecules from that given by the regular solution model:

$$
k_{i j}=\frac{E_{i j}-\left(\delta_{i}-\delta_{j}\right)^{2}}{2 \delta_{i} \delta_{j}} .
$$

The regular solution model applies to mixtures where molecules are of similar size and interact only through dispersion forces [17]. For mixtures of molecules of different size or where other forces are present (e.g., hydrogen bonding, dipole-dipole interactions, etc.), deviations from this model are to be expected. In this work, this is captured by the mixing parameter $k_{i j}$.

\subsection{Group contribution method}

In order to obtain accurate results with a cubic EOS, appropriate values for binary interaction parameters are required. Typically, the $k_{i j}$ 's are used as fit parameters used to reproduce experimental data. However, frequently the experimental data required to develop and validate the thermodynamic models are lacking. Several empirical methods have been proposed to estimate binary interaction parameters; however, many of these correlations fail to properly predict the phase behavior at elevated pressures [29].

Alternate mixing rules to the van der Waals mixing rule (see Eqs. (5) and (6)) have been proposed as in order to improve the accuracy of EOS's. One class of these is based on combining the EOS with an activity coefficient model [21] and is typically referred to as $\mathrm{EOS} / g^{E}$.

The use of group contribution techniques with activity coefficient models, such as UNIFAC, has been quite successful [14]. Calculating an EOS's parameters based on a group contribution method (GCM) is often more powerful than the use of activity coefficient models and can provide accurate predictions $[18,21]$. The combination of an EOS with a group contribution method results in a predictive model that provides a theoretical expression for $k_{i j}$

The interaction free energy energy $E_{i j}$ between a molecule of type $i$ and a molecule of type $j$, which appears in Eq. (20), can be expressed in terms of a sum of the interactions between pairs of groups within the molecules [20]

$$
E_{i j}=-\frac{1}{2} \sum_{k, l}\left(\alpha_{i k}-\alpha_{j k}\right)\left(\alpha_{i l}-\alpha_{j l}\right) A_{k l}(T)
$$

where the indices $k$ and $l$ run over all types of groups in the system, and $\alpha_{i k}$ is the fraction of molecule $i$ occupied by group $k$. For example, propane has a molecular structure of $\mathrm{CH}_{3}-\mathrm{CH}_{2}-\mathrm{CH}_{3}$; It contains two $\mathrm{CH}_{3}$ groups and one $\mathrm{CH}_{2}$ group. Therefore the total number of groups present in this molecule is three. In this case, the fraction of molecule propane occupied by group $\mathrm{CH}_{2}$ is $\alpha_{\text {propane-CH2 }}=1 / 3$, and the fraction of molecule propane occupied by group $\mathrm{CH}_{3}$ is $\alpha_{\text {propane-CH3 }}=2 / 3$. 
The temperature dependence of the interaction parameter $A_{k l}(T)$ is given by:

$$
A_{k l}(T)=A_{k l}^{0}\left(\frac{T_{0}}{T}\right)^{B_{k l}^{0} / A_{k l}^{0}-1}
$$

where $T$ is the absolute temperature, $T_{0}=298.15 \mathrm{~K}$ is a reference temperature, and $A_{k l}^{0}$ and $B_{k l}^{0}$ are the interaction parameters between groups $k$ and $l$.

The quantity $A_{k l}(T)$ represents the negative free energy of interaction between a group of type $k$ and a group of type $l$. From the Gibbs-Helmholtz equation [17], we can then identify the quantity

$$
B_{k l}^{0}\left(\frac{T_{0}}{T}\right)^{B_{k l}^{0} / A_{k l}^{0}-1}
$$

with the attractive energy of interaction between groups, and, consequently, the quantity

$$
A_{k l}^{0}\left(B_{k l}^{0} / A_{k l}^{0}-1\right)\left(\frac{T_{0}}{T}\right)^{B_{k l}^{0} / A_{k l}^{0}-1}
$$

is related with the entropy of the interaction.

This group contribution method (GCM) was used by Jaubert and Noël [21] to predict the VLE of several binary mixture of hydrocarbon components using the Peng-Robinson EOS (PR), calling this the predictive Peng-Robinson 1978 (PPR78). Noël showed that the obtained results from GCM are often more precise than $\mathrm{EOS} / g^{E}$ models. Another study used the GCM to predict $k_{i j}$ of a system containing hydrocarbon components and carbon dioxide $\mathrm{CO}_{2}$ using SRK EOS [23]. The study indicated its feasibility to estimate the $k_{i j}$ of any mixture containing carbon dioxide and hydrocarbons at any temperature. A relation between the $k_{i j}$ parameters for PPR78 and the SRK EOS has been developed [22]. This helps to predict GCM parameters of SRK EOS based on PR EOS GCM parameters. Consequently, the values for the group interaction parameters $A_{k l}^{0}$ and $B_{k l}^{0}$ between a large number of different types of groups is already available.

\section{Results and discussion}

In this section, we determine the values of the group interaction parameters $A_{k l}^{0}$ and $B_{k l}^{0}$ appearing in Eq. (23) between elemental mercury and various molecular groups. As an initial step in this process, we need to ensure that the properties of the pure components are properly described by the SRK equation of state. This is done by ensuring the vapor pressure curves are accurately reproduced, which is described in the next section.
Once the pure component parameters of the SRK EOS are chosen, the values of the group interaction parameters are determined by fitting experimental solubility data for mercury in a variety of solvents. This is done by minimizing the objective function $F_{\text {obj }}$

$$
F_{\mathrm{obj}}=\sum_{i}\left(\frac{S_{i}^{\text {calc }}-S_{i}^{\exp }}{S_{i}^{\exp }}\right)^{2}
$$

where $S_{i}^{\exp }$ is the experimental solubility of mercury in the selected solvent, and $S_{i}^{\text {calc }}$ is the calculated solubility of mercury in the selected solvent.

Vapor-liquid equilibrium (VLE) and liquid-liquid equilibrium (LLE) calculations were performed for the SRK equation of state using standard flash algorithms implemented in Python to obtain the solubility of mercury $S_{i}^{\text {calc }}$. The LmFit package in Python was used to determine the values of the group interaction parameters $A_{k l}^{0}$ and $B_{k l}^{0}$ in Eq. (23) that minimize the objective function. The optimized values and their uncertainties are summarized in Table 1. These are discussed in more detail in the following parts of this section.

Table 1: Group interaction parameters $A^{0}$ and $B^{0}$ for mercury with other groups.

\begin{tabular}{lrrr}
\hline \hline group & $\begin{array}{r}10^{-4} A^{0} \\
\text { bar }^{-1}\end{array}$ & $\begin{array}{r}10^{-4} B^{0} \\
\text { bar }^{-1}\end{array}$ & $\begin{array}{r}B^{0} / A^{0} \\
-\end{array}$ \\
\hline $\mathrm{CH}$ & $10.9143 \pm 0.0023$ & $7.00 \pm 0.0945$ & 0.66 \\
$\mathrm{CH}_{2}$ & $7.8864 \pm 0.0057$ & $7.0562 \pm 0.065$ & 0.89 \\
$\mathrm{CH}_{3}$ & $8.5137 \pm 0.0207$ & $7.1461 \pm 0.27$ & 0.84 \\
$\mathrm{OH}$ & $6.5524 \pm 0.00204$ & $5.29903 \pm 0.837$ & 0.80 \\
$\mathrm{ACH}$ & $7.7506 \pm 0.0036$ & $8.1350 \pm 0.01$ & 1.049 \\
$\mathrm{ACCH}$ & $7.5699 \pm 0.028$ & $7.9629 \pm 1.1$ & 1.052 \\
$\mathrm{H}_{2} \mathrm{O}$ & $9.9037 \pm 0.0063$ & $3.7305 \pm 0.0289$ & 0.38 \\
\hline \hline
\end{tabular}

\subsection{Vapor pressure}

In order to predict the pure component properties of a species, the SRK equation of state requires its critical pressure, critical temperature and acentric factor. In principle, these can be obtained

In the literature, pure fluid parameters vary slightly from reference to reference. In this work, the acentric factor was only tuned in order to achieve the minimum absolute average relative deviation error (AARD) in vapor pressure. The adjusted acentric factor and critical pressure, critical temperature are summarized in Table 2 . Note that these values are in good agreement with the accepted experimental critical properties for these compounds in the literature. 
Figure 1 indicates that the SRK EOS is capable of accurately predicting the vapor pressure of elemental mercury, water, and alcohols. The AARD for 99 experimental data points of elemental mercury over a temperature range of $253.15 \mathrm{~K}$ to $773.15 \mathrm{~K}$ was $3.7 \%$, the experimental data used in this work were taken from Refs. 30, 31, 32, 33. These data were classified by $\mathrm{Hu}-$ ber et al. as primary experimental data, because of their low experimental uncertainty of around $1 \%$ [12].

The AARD for the vapor pressure of water was $2.5 \%$ for 38 experimental data points over a temperature range of $319.6 \mathrm{~K}$ to $449.7 \mathrm{~K}$; the experimental data used in this work were taken from Ref. 34. For methanol and isopropanol, the AARD was $2.8 \%$ for 24 data points and $2.6 \%$ for 14 experimental data points, respectively; the experimental data were taken from Ref. 35, 36, 37.

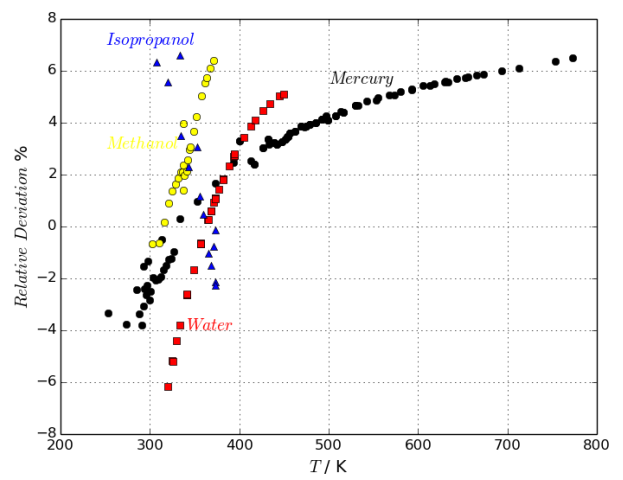

Figure 1: Relative deviation in vapor pressure for elemental mercury (black), water (red), methanol, (yellow), and isopropanol (blue).

Figure 2 shows the relative deviation of the predictions of the SRK equation of state for the vapor pressure of some $n$-alkanes and aromatic compounds. The AARD for propane, $n$-pentane, and $n$-decane was $0.4 \%$ for 31 experimental data points, $0.2 \%$ for 50 experimental data points, and $2.5 \%$ for 32 experimental data points respectively. the experimental vapor pressure data were taken from Refs. 38, 39, and 40. In addition, the AARD for the vapor pressure of benzene, toluene, and $o$-xylene was $0.9 \%$ for 13 experimental data points, $0.3 \%$ for 17 data points, and $0.78 \%$ for 12 data points, respectively; the experimental vapor pressure data for aromatics were taken from Refs. 41,42, and 43.

\subsection{Solubility of mercury in water}

The solubility of elemental mercury in water is available over a wide range of temperatures. The experimental data used in this work were taken from Ref. 47,
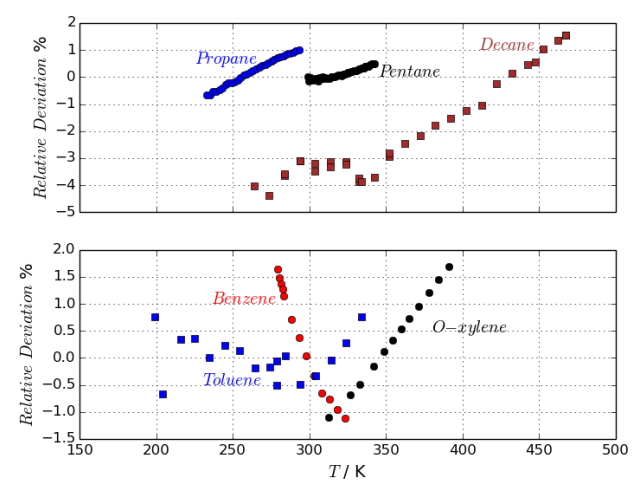

Figure 2: Relative deviation in vapor pressure of (a) $n$-alkanes and (b) aromatic compounds.

which are shown as the symbols in Fig. 3(a) over a temperature range of $273.15 \mathrm{~K}$ to $393.15 \mathrm{~K}$. The dashed line in Fig. 3(a) is the solubility of mercury predicted by the SRK EOS, neglecting the binary interaction parameter (i.e. $k_{i j}=0$ ); without introducing proper binary interaction parameters, the mercury solubility in water is severely overestimated. The solid line in Fig. 3(a) gives the prediction of the SRK EOS with the $k_{i j}$ shown in Fig. 3(b). For this system, an AARD of $4.2 \%$ was obtained for 25 experimental data points. The binary interaction parameter between mercury and water is temperature dependent; it increases by 0.05 with each $20 \mathrm{~K}$ increase in temperature.
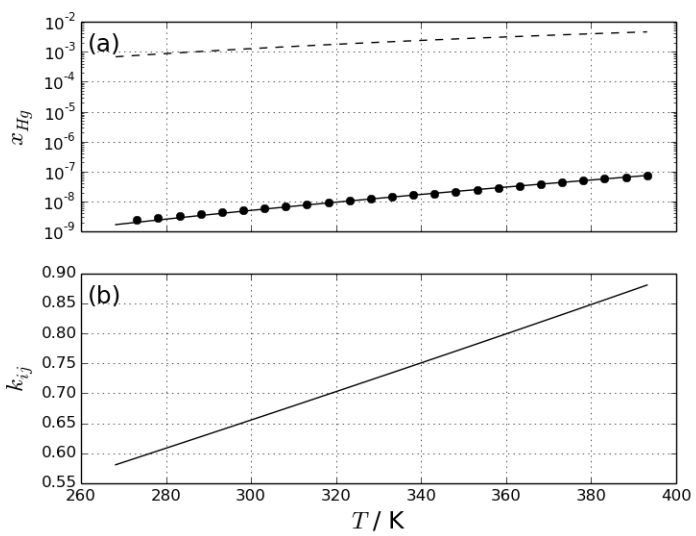

Figure 3: (a) Solubility of mercury in water. The dashed line is the prediction of the SRK EOS with $k_{i j}=0$, and the solid line is the prediction with $k_{i j}$ estimated using the group contribution method. (b) The variation with temperature of the binary interaction parameter between mercury and water. 
Table 2: Pure component critical properties and acentric factor.

\begin{tabular}{lrrrr}
\hline \hline Component & $\begin{array}{r}p_{c} \\
\text { bar }\end{array}$ & $T_{c}$ & $\omega$ & Ref. \\
& 1670.0 & 1764 & -0.2102 & {$[44]$} \\
\hline mercury & 42.55 & 369.92 & 0.152 & {$[45]$} \\
propane & 37.966 & 425.16 & 0.205 & {$[45]$} \\
$n$-butane & 33.691 & 469.7 & 0.250 & {$[45]$} \\
$n$-pentane & 30.124 & 507.31 & 0.305 & {$[45]$} \\
$n$-hexane & 27.358 & 540.1 & 0.3525 & {$[45]$} \\
$n$-heptane & 24.865 & 568.76 & 0.3978 & {$[45]$} \\
$n$-octane & 22.879 & 594.56 & 0.4419 & {$[45]$} \\
$n$-nonane & 21.035 & 617.5 & 0.492 & {$[45]$} \\
$n$-decane & 48.98 & 562.79 & 0.2130 & {$[45]$} \\
benzene & 43.2 & 591.8 & 0.268 & {$[45]$} \\
toluene & 39.8 & 633.3 & 0.304 & {$[45]$} \\
$o$-xylene & 80.959 & 512.5 & 0.556 & {$[45]$} \\
methanol & 47.63 & 508.37 & 0.657 & {$[45]$} \\
isopropanol & 220.64 & 647.14 & 0.324 & {$[46]$} \\
water & & & & \\
\hline \hline
\end{tabular}

Thermodynamically, the ratio $B^{0} / A^{0}$ reflects the influence of entropy on the mixing of groups. If the ratio is less than one, the mixing process tends to increase entropy; the molecules become more disordered than in ideal mixing. If the ratio is greater than one, then entropy is lost in mixing; the molecules are more ordered than in ideal mixing. For a ratio of one, there is no excess entropy of mixing and, and enthalpy drives the process. In this case, the binary interaction parameter temperature independent.

\subsection{Solubility of mercury in normal alkanes}

Normal alkanes represent more than $90 \%$ of natural gas and crude oil species. Predicting mercury solubility in these species is crucial. Elemental mercury is considered the dominant mercury species in the crude oil and natural gas $[9,48]$. The solubility data of elemental mercury in hydrocarbon systems are sparse and covers a limited temperature range, The experimental data used in this work are shown as the symbols in Fig. 4(a) and (b) for alkanes from $\mathrm{C}_{5}$ to $\mathrm{C}_{10}$, and Fig. 5(a) and (b) for $\mathrm{C}_{3}$ and $\mathrm{C}_{4}$. These data were taken from Ref. 49 and Refs. 50 . Around 65 experimental data points for $\mathrm{C}_{5}$ to $\mathrm{C}_{10}$ over a temperature range of $273.15 \mathrm{~K}$ to $336.15 \mathrm{~K}$ and atmospheric pressure, and 3 experimental data points for $\mathrm{C}_{8}$ over a temperature range from $338.15 \mathrm{~K}$ to $473.15 \mathrm{~K}$ and 6 bar. In addition to 17 data points for $\mathrm{C}_{3}$ and $\mathrm{C}_{4}$ at different temperatures and pressures.
Figures 4(a) and (b) show the predicted solubility of elemental mercury in normal alkanes from $\mathrm{C}_{5}$ to $\mathrm{C}_{10}$. The dashed lines in Fig. 4 are the solubilities predicted by the SRK EOS, neglecting the binary interaction parameter (i.e. $k_{i j}=0$ ); without introducing the proper binary interaction parameters, the mercury solubility in alkanes is nearly independent of the molecular weight of the alkanes. By introducing the binary interaction parameter, the results indicated by the solid lines in Fig. 4 are obtained. The AARD for the solubility in normal alkanes from $C_{3}$ to $C_{10}$ was $5.47 \%$ for 74 experimental data points.

In the recent study of Polishuk et al. [19], the PengRobinson (PR) and PC-SAFT equations of state were used to predict the properties of mercury-hydrocarbon mixtures. In their work, a single, constant value of $k_{i j}$, which was fixed by fitting to experimental solubility data of mercury in $n$-pentane, was used. The results of the study showed that within this approach, the PengRobinson EOS was incapable of estimating the solubility of mercury in the studied hydrocarbon systems, apart from mercury-pentane. The results presented in Fig. 3 of Polishuk et al. show that the predicted solubility of mercury in $\mathrm{C}_{8}$ using PC-SAFT and the PR $\mathrm{EoS}$ at $298.15^{\circ} \mathrm{C}$ was $0.91 \mathrm{ppm}$ and $3.5 \mathrm{ppm}$, respectively, while the experimental solubility was $1.08 \mathrm{ppm}$. The value obtained in this study using the GCM was $1.10 \mathrm{ppm}$ which is much closer to the experimental value.

In our study, different $k_{i j}$ values were calculated using GCM for each mercury-hydrocarbon binary system at the system temperature and pressure. This approach improves the prediction of mercury solubility in normal alkanes more accurately than fixing $k_{i j}$ to a single value. The solubility of elemental mercury increases with the carbon numbers, which is in consistent with the observations of Refs. 49 and 50.

Several process facilities, such as stripping columns, heat exchangers, reactors, and distillation units operate at high temperatures; therefore, predicting mercury solubility in alkanes at high temperature is crucial. The solubility of elemental mercury in some organic solvents, including octane, dodecane, and toluene, has been experimentally and theoretically estimated over a temperature range from $100^{\circ} \mathrm{C}$ to $200^{\circ} \mathrm{C}$ and up to 6 bar [50]. Figure 4(b) represents the predicted solubility of elemental mercury in normal octane at 6 bar and high temperatures.

Figures $5(a)$ and $(b)$ show the solubility of mercury in propane and butane, respectively, at a range of pressures and temperatures. It is clear that the SRK EOS predicts the solubility of elemental mercury in light hy- 


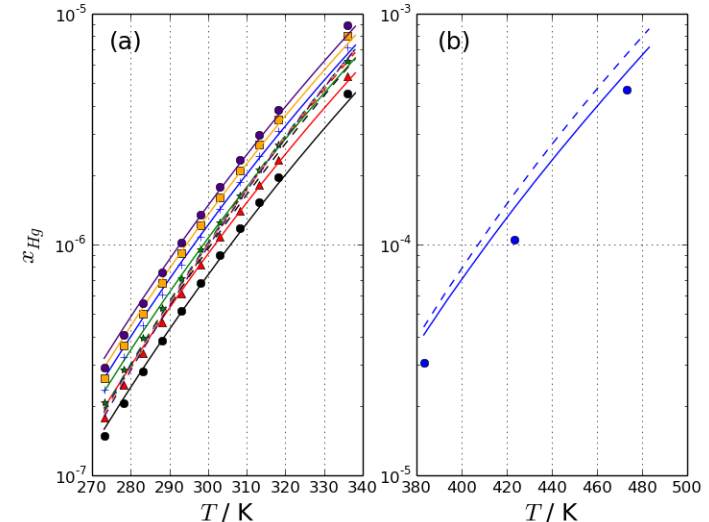

Figure 4: Solubility of mercury in normal alkanes: $\mathrm{C}_{5}$ (black), $\mathrm{C}_{6}$ (red), $\mathrm{C}_{7}$ (green), $\mathrm{C}_{8}$ (blue), $\mathrm{C}_{9}$ (orange), and $\mathrm{C}_{10}$ (indigo). The symbols represent experimental data, the solid lines represent predicted solubility with the binary interaction parameters estimated using the GCM, and the dashed lines represent the solubility without introducing the binary interaction parameter.

drocarbons well. This is due to fact that cubic EOS's are capable of predicting vapor phase properties more accurately than liquid phase properties. It can be noticed that the solubility of elemental mercury in propane is almost equal to that in butane. This implies that the solubility of mercury in light hydrocarbons in the gas phase is independent of carbon number. This suggests that the interaction of elemental mercury with methane or ethane is similar to that with propane and butane. This enables the estimation of mercury solubility in methane, as the experimental data are unavailable.

The binary interaction parameters of mercury in normal alkanes from $\mathrm{C}_{5}$ to $\mathrm{C}_{10}$ are shown in Fig. 6 . The interaction of mercury with these higher molecular weight alkanes depends on both the carbon number and temperature.

\subsection{Solubility of mercury in aromatics}

Aromatics are considered to be the main raw material for many petrochemical industries [51]. The naphtha reforming process is one of main sources of aromatics. As crude oil and natural gas are the main sources of aromatics and crude oil is known to contain mercury, predicting the solubility of mercury in aromatics is vital of importance.

Figure 7(a) shows the solubility of elemental mercury in benzene, toluene, and $o$-xylene over a range of temperatures. The experimental data are taken from Ref. 49, which are shown as the symbols. The dashed lines are the predictions of the SRK EOS with $k_{i j}=0$.
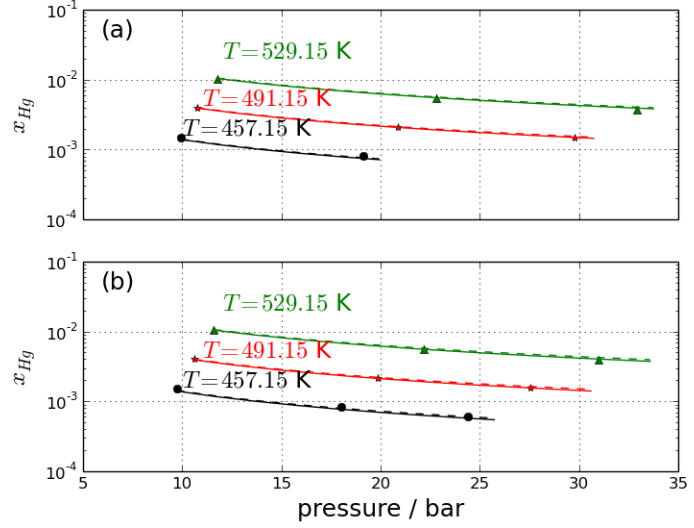

Figure 5: Solubility of mercury in (a) propane and (b) butane.

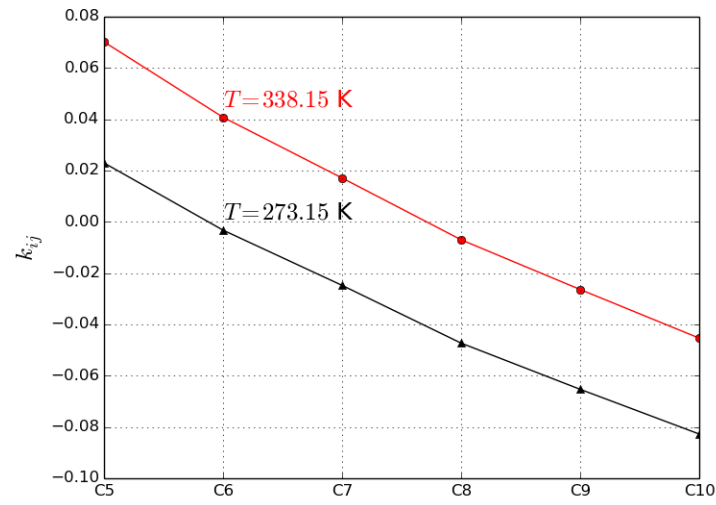

Figure 6: Binary interaction parameter for mercury-alkane mixtures.

It is clear that by neglecting the binary interaction parameters, the predicted solubility of elemental mercury in aromatics is relatively insensitive to the presence of methyl groups.

Two types of interaction groups for benzene, toluene, and $o$-xylene were defined by dividing the carbons in the aromatic ring. One group $\mathrm{ACH}$ is an aromatic carbon that is attached to a hydrogen atom; benzene has six of these groups, while toluene has only five and $o$ xylene has four. The other group $\mathrm{ACCH} 3$ is an aromatic carbon attached to a methyl group; benzene has none of these groups, toluene has one, and $o$-xylene has two. The values of the interaction parameters of these groups with elemental mercury were fit to the solubility data for mercury in benzene and in $o$-xylene. These are summarized in Table 1. 

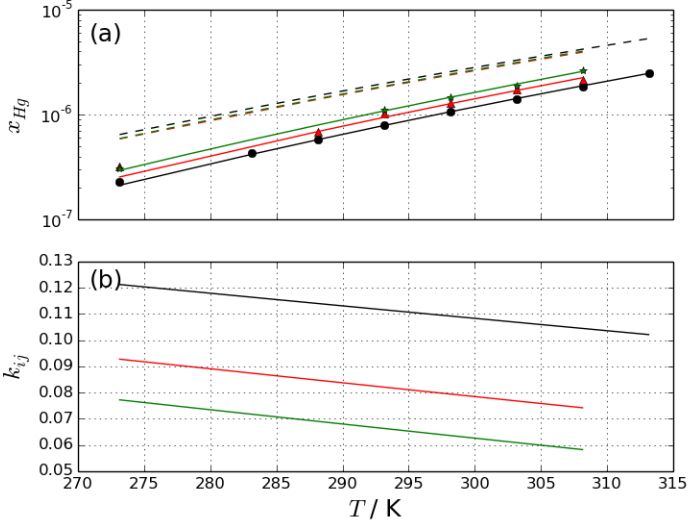

Figure 7: (a) Solubility and (b) binary interaction parameter of mercury with in benzene (black), toluene (red), and $o$-xylene (green).

The solid lines in Fig. 7(a) show the solubilities calculated by the SRK EOS with the binary interaction parameters estimated by the group contribution method. As a test of the group contribution model, the binary interaction parameter between mercury and toluene was predicted based on the group interaction parameters obtained from mercury-benzene and mercury- $o$-xylene mixtures. The AARD for mercury in benzene, toluene, and $o$-xylene was $1.87 \%$ for 8 data points over a temperature range of $273.15 \mathrm{~K}$ to $313.15 \mathrm{~K}, 6.1 \%$ for 6 data points over a temperature range of $273.15 \mathrm{~K}$ to $308.15 \mathrm{~K}$, and $2.7 \%$ for 5 data points over a temperature range of $273.15 \mathrm{~K}$ to $308.15 \mathrm{~K}$ and atmospheric pressure, respectively.

The results presented in Fig. 4 of the Polishuk et al. [19] study show that the predicted solubility of mercury in toluene using PC-SAFT and PR EoS at $293.15^{\circ} \mathrm{C}$ was $0.91 \mathrm{ppm}$ and $1.05 \mathrm{ppm}$, respectively, while the experimental solubility was $0.98 \mathrm{ppm}$. The value obtained in this work using the GCM and based on the group interaction parameters obtained from mercury-benzene and mercury- $o$-xylene mixtures was $0.94 \mathrm{ppm}$, which better reflects the experimental value. The GCM is capable of predicting binary interaction parameters of compounds where experimental data are unavailable.

By introducing binary interaction parameters, the solubility of elemental mercury in aromatics is found to increase with the number of methyl groups, which is consistent with what is experimentally observed. Figure 7(b) indicates that the interaction between mercury and aromatics is fairly independent of temperature.

\subsection{Solubility of mercury in alcohols}

Alcohols such as mono-ethylene glycol (MEG) and diethylene glycol (DEG) are widely used in oil and gas processing as anti-freeze and anti-corrosion agents; however, experimental data for the solubility of mercury in these alcohols are not available in the literature. One of the motivations of this work is to predict mercury solubility in such alcohols.

Experimental data are available for the solubility of mercury in methanol and isopropanol [47]. Figure 8(a) shows a comparison of the SRK EOS, with and without the binary interaction parameter, and experimental measurements for the solubility of mercury in methanol and isopropanol. Significant deviation can be observed between the experimental data and correlated results when $k_{i j}=0$.

The group interaction parameters between elemental mercury and the $\mathrm{OH}$ group were determined by fitting experimental solubility data for alcohols (see Table 1). Figure 8(b) shows that the $k_{i j}$ between mercury and isopropanol is more temperature dependent than methanol.

Using the group contribution method, the interaction between mercury and MEG or DEG can be easily predicted. As a test of the group contribution model, we predict the solubility of mercury in MEG. Large quantities of MEG are injected at the wellhead in order to avoid hydrate formation during transportation process. The partitioning of elemental mercury from a gas phase into MEG solutions was investigated under standard laboratory conditions [52]. It was observed that the solubility of elemental mercury in MEG ranged from 0 to $60 \mathrm{ppb}$. Using the SRK combined with GCM developed in this work to estimate $k_{i j}$, we predict that the solubility of mercury in MEG is $57.7 \mathrm{ppb}$. Using $k_{i j}=0$, the solubility of mercury in MEG is $1.78 \mathrm{ppm}$. It is clear that the SRK combined with the GCM is able to predict mercury solubility in alcohol systems.

\section{Conclusions}

Mercury is not only a toxic pollutant in the environment, but it is also a corrosive element to processing equipment. Understanding mercury pathways in an ecosystem or its distribution in process facilities requires a model that is able to predict its thermodynamic behavior in a wide variety of conditions and solvents. In this work, we parameterize a group contribution method to estimate the temperature dependent binary interaction parameters between elemental mercury and compounds composed of $\mathrm{CH}, \mathrm{CH}_{2}, \mathrm{CH}_{3}, \mathrm{OH}, \mathrm{H}_{2} \mathrm{O}, \mathrm{ACH}$ and $\mathrm{ACCH}_{3}$ groups. By using these binary interaction 

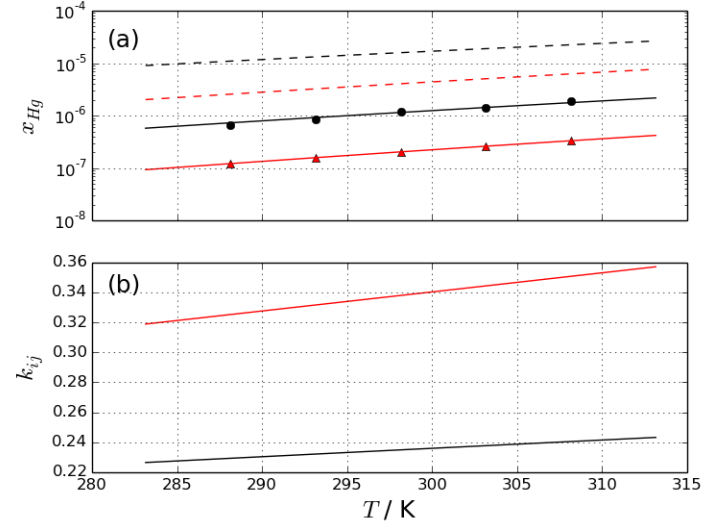

Figure 8: Solubility of mercury in methanol (black) and isopropanol (red). The symbols represent experimental data, the solid lines are predicted solubilities with the binary interaction parameter estimated using the GCM, and the dashed lines are predictions with the binary interaction parameter set to zero.

parameters, we find that the SRK EOS provides a good description of mercury solubility in water, alkanes, alcohols, and aromatic solvents, as compared to available experimental data. Improper estimates for $k_{i j}$ can yield extremely poor results; for instance, setting $k_{i j}=0$, the SRK predicts that the solubility of mercury in water at $298 \mathrm{~K}$ is 3,374 ppm, compared to the experimental value of less than $1 \mathrm{ppm}$. The group contribution method allows the estimation of $k_{i j}$ of elemental mercury with a wide variety of solvents and solvent mixtures, even when experimental data are not available. The group interaction parameters are already available for a wide range of systems [21, 23, 22], making this approach immediately usable in practical applications.

Currently, we are using this group contribution model to estimate the partitioning of elemental mercury through a gas processing facility and validating the calculations against field data. In future, we intend to extend this approach to organic mercury compounds to examine the speciation and distribution of mercury.

\section{Acknowledgments}

MK is grateful to the Department of Chemical and Process Engineering Department at the University of Strathclyde and to the Libyan Ministry of Higher Education and Scientific Research for financial support. The authors are thankful to Dr. Karen Johnston for useful discussions.

\section{References}

[1] A. Maulvault, P. Anacleto, V. Barbosa, J. J. Sloth, R. Rasmussen, A. Tediosi, M. Fernandez-Tejedor, F. H. van den Heuvel, M. Kotterman, A. Marques, Toxic elements and speciation in seafood samples from different contaminated sites in Europe, Environ. Res. 143, Part B (2015) 72 - 81. doi:10.1016/j.envres.2015.09.016.

[2] H. D. Choi, J. Huang, S. Mondal, T. M. Holsen, Variation in concentrations of three mercury $(\mathrm{Hg})$ forms at a rural and a suburban site in New York State, Sci. Total Environ. 448 (2013) 96 - 106. doi:10.1016/j.scitotenv.2012.08.052.

[3] C. Deng, D. Zhang, X. Pan, F. Chang, S. Wang, Toxic effects of mercury on $\{\mathrm{PSI}\}$ and $\{\mathrm{PSII}\}$ activities, membrane potential and transthylakoid proton gradient in microsorium pteropus, J. Photochem. Photobiol. B 127 (2013) 1 - 7. doi:10.1016/j.jphotobiol.2013.07.012.

[4] R. Ferrara, B. Mazzolai, E. Lanzillotta, E. Nucaro, N. Pirrone, Volcanoes as emission sources of atmospheric mercury in the Mediterranean basin, Sci. Total Environ. 259 (1-3) (2000) 115 121. doi:10.1016/S0048-9697(00)00558-1.

[5] Q. Liu, Mercury concentration in natural gas and its distribution in the Tarim Basin, Sci. China Earth Sci. 56 (8) (2013) 13711379. doi:10.1007/s11430-013-4609-2.

[6] F. Wang, S. Wang, L. Zhang, H. Yang, Q. Wu, J. Hao, Mercury enrichment and its effects on atmospheric emissions in cement plants of China, Atmospheric Environment 92 (2014) 421 - 428. doi:10.1016/j.atmosenv.2014.04.029.

[7] N. Pirrone, S. Cinnirella, X. Feng, R. B. Finkelman, H. R. Friedli, J. Leaner, R. Mason, A. B. Mukherjee, G. B. Stracher, D. G. Streets, K. Telmer, Global mercury emissions to the atmosphere from anthropogenic and natural sources, Atmos. Chem. Phys. 10 (2010) 5951-5964. doi:10.5194/acp-10-5951-2010.

[8] S. M. Wilhelm, Avoiding exposure to mercury during inspection and maintenance operations in oil and gas processing, Process Saf. Prog. 18 (3) (1999) 178-188. doi:10.1002/prs.680180311.

[9] S. M. Wilhelm, N. Bloom, Mercury in petroleum, Fuel Processing Technology 63 (1) (2000) 1 - 27. doi:10.1016/S03783820(99)00068-5.

[10] L. D. Hylander, M. E. Goodsite, Environmental costs of mercury pollution, Sci. Total Environ. 368 (1) (2006) 352 - 370. doi:10.1016/j.scitotenv.2005.11.029.

[11] G. J. F. Holman, C. A. ten Seldam, A critical evaluation of the thermophysical properties of mercury, J. Phys. Chem. Ref. Data 23 (5) (1994) 807-827. doi:10.1063/1.555952.

[12] M. L. Huber, A. Laesecke, D. G. Friend, Correlation for the vapor pressure of mercury, Ind. Eng. Chem. 45 (2006) 73517361. doi:10.1021/ie060560s.

[13] A. Merlone, C. Musacchio, The mercury vapour pressure vs. temperature relation between $(500$ and 665$) \mathrm{K}$, J. Chem. Thermodyn. 42 (1) (2010) 38 - 47. doi:10.1016/j.jct.2009.07.012.

[14] C.-C. Chen, P. M. Mathias, Applied thermodynamics for process modeling, AIChE J. 48 (2) (2002) 194-200. doi:10.1002/aic.690480202.

[15] M. H. Abraham, J. Gil-Lostes, W. E. Acree, Jr, J. Enrique Cometto-Muniz, W. S. Cain, Solvation parameters for mercury and mercury(ii) compounds: calculation of properties of environmental interest, J. Environ. Monit. 10 (2008) 435-442. doi:10.1039/B719685G.

[16] M. H. Lagache, J. Ridard, P. Ungerer, A. Boutin, Force field optimization for organic mercury compounds, J. Phys. Chem. B 108 (24) (2004) 8419-8426. doi:10.1021/jp049676x.

[17] J. M. Prausnitz, R. N. Lichtenthaler, E. G. de Azevedo, Molecular Thermodynamics of Fluid-Phase Equilibria, 2nd Edition, Prentice-Hall, Englewood Cliffs, NJ, 1986. 
[18] M. B. Ewing, C. J. Peters, Fundamental considerations, in: C. P. J.V. Sengers, R.F. Kayser, H. White (Eds.), Equations of State for Fluids and Fluid Mixtures, Vol. 5 of Experimental Thermodynamics, Elsevier, 2000, pp. 5 - 34. doi:10.1016/S18745644(00)80013-2.

[19] I. Polishuk, F. Nakonechny, N. Brauner, Predicting phase behavior of metallic mercury in liquid and compressed gaseous hydrocarbons, Fuel 174 (2016) 197 - 205. doi:10.1016/j.fuel.2016.02.002.

[20] A. Peneloux, W. Abdoul, E. Rauzy, Excess functions and equations of state, Fluid Phase Equilib. 47 (23) (1989) 115 - 132. doi:10.1016/0378-3812(89)80172-4.

[21] J.-N. Jaubert, F. Mutelet, VLE predictions with the PengRobinson equation of state and temperature dependent $k_{i j}$ calculated through a group contribution method, Fluid Phase Equilib. 224 (2) (2004) 285 - 304. doi:10.1016/j.fluid.2004.06.059.

[22] J.-N. Jaubert, R. Privat, Relationship between the binary interaction parameters $\left(k_{i j}\right)$ of the Peng-Robinson and those of the Soave-Redlich-Kwong equations of state: Application to the definition of the PR2SRK model, Fluid Phase Equilib. 295 (1) (2010) 26 - 37. doi:10.1016/j.fluid.2010.03.037.

[23] N. Abedi, K. Nasrifar, Group contribution method for predicting the phase behavior of binary mixtures containing carbon dioxide, Iran. J. Chem. Eng. 9 (1) (2012) 12-22.

[24] O. Redlich, J. N. S. Kwong, On the thermodynamics of solutions. V. An equation of state. Fugacities of gaseous solutions., Chem. Rev. 44 (1) (1949) 233-244. doi:10.1021/cr60137a013.

[25] G. Soave, Equilibrium constants from a modified RedlichKwong equation of state, Chem. Eng. Sci. 27 (6) (1972) 1197 - 1203. doi:10.1016/0009-2509(72)80096-4.

[26] T. Kwak, G. Mansoori, Van der waals mixing rules for cubic equations of state, applications for supercritical fluid extraction modell, Chem. Eng. Sci. 41 (5) (1986) 1303-1309.

[27] D. S. H. Wong, S. I. Sandler, A theoretically correct mixing rule for cubic equations of state, AIChE J. 38 (5) (1992) 671-680. doi:10.1002/aic.690380505.

[28] G. K. F. Georgios M. Kontogeorgis, Thermodynamic Models for Industrial Applications From Classical and Advanced Mixing Rules to Association Theories, John Wiley and Sons Ltd, 2010.

[29] D. Minicucci, X.-Y. Zou, J. Shaw, The impact of liquid-liquidvapour phase behaviour on coke formation from model coke precursors, Fluid Phase Equilib. 194-197 (2002) 353 - 360. doi:10.1016/S0378-3812(01)00787-7.

[30] H. Ernsberger, F.M.;Pitmam, New absolute manometer for vapor pressure in the micron range, Rev. Sci. Instrum. 26 (4) (1955) 584-588. doi:doi.org/10.1063/1.1715251.

[31] D. Ambrose, C. H. S. Sprake, The vapor pressure of mercury, J. Chem. Thermodyn 4 (4) (1972) 603-620. doi:10.1016/00219614(72)90082-1.

[32] C. Merlone, A.;Musacchio, The mercury vapour pressure versus temperature relation between 500 and $665 \mathrm{k}$, J. Chem. Thermodyn. 42 (1) (2010) 38-47. doi:doi.org/10.1016/j.jct.2009.07.012.

[33] T. B. Douglas, A. F. Ball, D. C. Ginnings, Heat capacity of liquid mercury between $0^{\circ}$ and $450^{\circ} \mathrm{C}$; Calculation of certain thermodynamic properties of the saturated liquid and vapor, J. Res. Natl. Bur. Stand. 46 (4) (1951) 2204.

[34] D. Ambrose, M. B. Ewing, N. B. Ghiassee, J. C. S. Ochoa, The ebulliometric method of vapor-pressure measurement: Vapor pressures of benzene, hexafluorobenzene, and naphthale, J. Chem. Thermodyn. 22 (6) (1990) 589-605. doi:10.1016/00219614(90)90151-F

[35] C. S. Moreira Gomes, H. N. M. de Oliveira, O. ChiavoneFilho, E. L. Foletto, Vapor-liquid equilibria for ethyl acetate + methanol and ethyl acetate + ethanol mixtures: Experimental verification and prediction, Chem. Eng. Res. Des. 92 (19) (2014) 2861-2866. doi:10.1016/j.cherd.2014.07.010.

[36] D. S. M. Constantino, C. S. M. Pereira, S. a. P. Pinho, V. M. T. M. Silva, A. E. Rodrigues, Isobaric vaporliquid equilibrium data for binary system of glycerol ethyl acetal and acetonitrile at $60.0 \mathrm{kpa}$ and $97.8 \mathrm{kpa}$, J. Chem. Eng. Data 58 (6) (2013) 17171723. doi: $10.1021 / \mathrm{je} 400138 \mathrm{~m}$.

[37] S. A. Iwarere, J. D. Raal, P. Naidoo, D. Ramjugernath, Vapourliquid equilibrium of carboxylic acid-alcohol binary systems: 2propanol + butyric acid, 2-butanol + butyric acid and 2-methyl1-propanol + butyric acid, Fluid Phase Equilib. (2014) 1827doi:10.1016/j.fluid.2014.07.025.

[38] X. Dong, M. Gong, J. Liu, J. Wu, Experimental measurement of vapor pressures and (vapor + liquid) equilibrium for 1,1,1,2tetrafluoroethane (R134a) + propane (R290) by a recirculation apparatus with view windows, J. Chem. Thermodyn. 43 (3) (2011) 505 - 510. doi:10.1016/j.jct.2010.11.001.

[39] R. Rós, J. Ortega, L. Fernández, I. de Nuez, J. Wisniak, Improvements in the experimentation and the representation of thermodynamic properties iso- $p$ VLE and $y^{E}$ of alkyl propanoate + alkane binaries, J. Chem. Eng. Data 59 (1) (2014) 125-142. doi:10.1021/je4009415.

[40] C. Viton, M. Chavret, E. Behar, J. Jose, Vapor pressure of normal alkanes from decane to eicosane at temperatures from $244 \mathrm{k}$ to $469 \mathrm{k}$ and pressures from 0.4 pa to $164 \mathrm{kp}$, ELDATA Int. Electron. J. Phys. Chem. Data 2 (1996) 215-224.

[41] M. Nicolae, F. Oprea, Vaporliquid equilibrium for the binary mixtures of dipropylene glycol with aromatic hydrocarbons: Experimental and regression, Fluid Phase Equilibria 370 (2014) 34 - 42. doi:10.1016/j.fluid.2014.03.003.

[42] R. Garriga, P. Perez, M. Gracia, Total vapor pressure and excess Gibbs energy for binary mixtures of 1,1,2,2tetrachloroethane or tetrachloroethene with benzene at nine temperatures, Fluid Phase Equilib. 227 (1) (2005) 79-86. doi:10.1016/j.fluid.2004.02.021.

[43] I. Mokbel, E. Rauzy, J. P. Meille, J. Jose, Low vapor pressures of 12 aromatic hydrocarbons. experimental and calculated data using a group contribution method, Fluid Phase Equilib. 147 (1998) 271-284. doi:10.1016/S0378-3812(98)00234-9.

[44] V. Kozhevnikov, D. Arnold, E. Grodzinskii, S. Naurzakov, Phase transitions and critical phenomena in mercury fluid probed by sound, Fluid Phase Equilibria 125 (1) (1996) 149 157. doi:10.1016/S0378-3812(96)03099-3.

[45] G. Liessmann, W. Schmidt, S. Reiffarth, Data compilation of the saechsische olefinwerke boehlen 1 .

[46] J. M. P. Bruce E. Poling, J. P., The Properties of Gases and Liquids, fifth edition Edition, McGraw-Hill,New York, 2000.

[47] H. L. Clever (Ed.), Mercury in Liquids, Compressed Gases, Molten Salts and Other Elements, IUPAC Solubility Data Series, Pergamon, Amsterdam, 1987. doi:10.1016/B978-0-08-0359359.50011-X.

[48] S. M. Wilhelm, L. Liang, D. Cussen, D. A. Kirchgessner, Mercury in crude oil processed in the United States (2004), Environ. Sci. Technol. 41 (13) (2007) 4509-4514. doi:10.1021/es062742j.

[49] H. L. Clever, M. Iwamoto, Solubility of mercury in normal alkanes, Ind. Eng. Chem. Res. 26 (2) (1987) 336-337. doi:10.1021/ie00062a026.

[50] M. M. Miedaner, A. A. Migdisov, A. E. WilliamsJones, Solubility of metallic mercury in octane, dodecane and toluene at temperatures between $100^{\circ} \mathrm{C}$ and $200^{\circ} \mathrm{C}$, Geochim. Cosmochim. Acta 69 (23) (2005) 5511 - 5516. doi:doi.org/10.1016/j.gca.2005.06.029.

[51] T. Kimura, N. Hata, K. Sakashita, S. Asaoka, Production of aromatics from heavier $n$-paraffins on hybrid cracking- 
reforming catalyst, Catal. Today 185 (1) (2012) 119 - 125. doi:10.1016/j.cattod.2011.09.027.

[52] Y. M. Sabri, S. J. Ippolito, J. Tardio, P. Morrison, S. K. Bhargava, Studying mercury partition in monoethylene glycol (MEG) used in gas facilities, Fuel 159 (2015) 917 - 924. doi:10.1016/j.fuel.2015.07.047. 